\title{
ACCELERATION MECHANISM OF VOID DEVELOPMENT IN CONCRETE RIPRAP SEAWALLS
}

\author{
Aykut Pamuk ${ }^{1}$, Yumi Okabe' and Shinji Sato
}

\begin{abstract}
Laboratory experiments have been conducted to investigate the acceleration mechanism of the void development in the backfill of a seawall. It is found that the rate of sand leakage is proportional to the air amount in the soil medium. Therefore, the larger void appears to accelerate the further enlargement of the void. Leakage of sand has been found to be closely related to the seepage flow due to the pressure gradient between the soil medium and the water. Therefore, propagation of the wave-induced pressure response influences the leakage of sand. Compressibility and permeability of soil medium have been found to be two significant parameters for the rate of leakage.
\end{abstract}

Keywords: failure of seawalls; sand leakage; pressure propagation; oscillatory flow tunnel

\section{INTRODUCTION}

Among the failure modes of the seawalls, the collapse of the filled area due to leakage of backfill material causes destruction on the landward side of the seawall. The general man-made structures constructed behind the seawalls such as roads, recreational areas or buildings are subjected to destruction because of this type of failure. Surfaces of backfills are usually paved with asphalt or concrete. The collapses of these pavements are generally sudden, without giving a warning. Therefore, the physical development of voids due to leakage of backfill causes threat for the living, as the areas behind seawalls are highly populated owing to the attractiveness of the coastal zone.

Through holes or tears on geotextile, sheet walls or filter layers, backfill material can flow out towards the seaside or towards armor layer. Wave pressures, forces or chemical deteriorations on a seawall can create these holes and tears. Also, during the construction stage, the integrity of geotextile can be spoiled during placement of large rocks.

In June 2015, a road and a sidewalk caved in just behind a seawall in Zonguldak, Turkey (Figure 1). It is necessary to see that there is no protection in front of the seawall where the collapse has occurred. Due to lack of protection, backfill material has been leaked easily towards sea side. However, natural rocks have prevented from such destruction at the other parts of the seawall. Thus, subsidence of road occurred just behind this section of the seawall. According to the interviews with the authorities, this part of the road has been collapsed 3 times in 10 years. One year later, the site visit to this location revealed that instead of putting stones in front of the seawall or protect the backfill material to discharge towards sea side, the municipality has decided to repair only the road and the pavement. Therefore, another collapse at this section of the road can be anticipated.
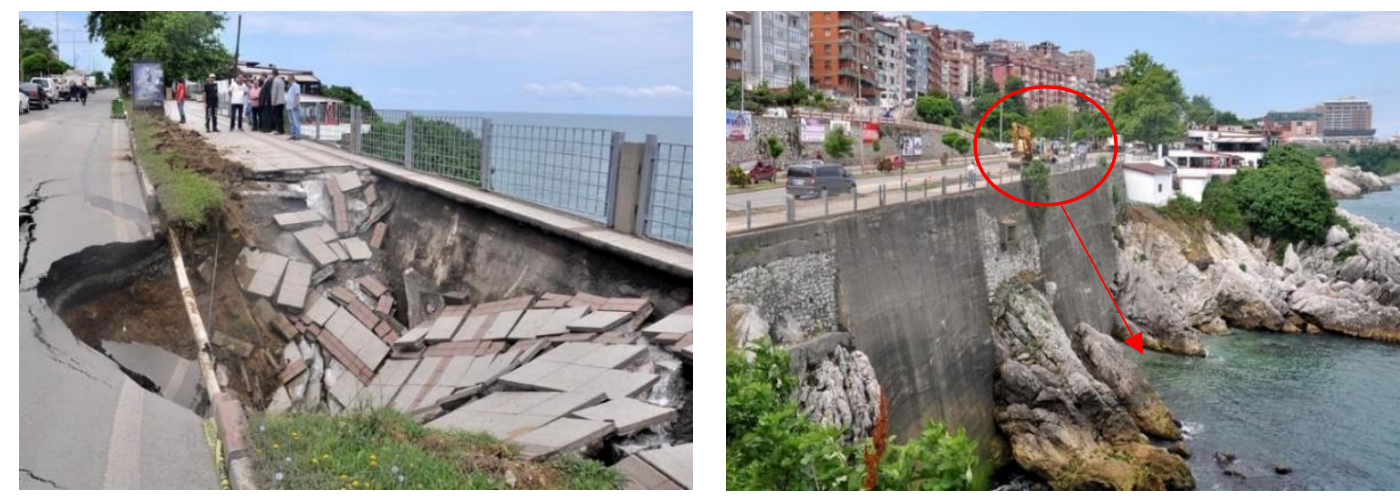

Figure 1 Example of collapse of pavement in Zonguldak, Turkey (CNN TURK, June 25, 2015)

Several studies have focused on especially the leakage of backfill material for the seawalls. For example, Takahashi et al. (1996) revealed the stages that lead to settlement of backfill pavement (Figure 2). According to the laboratory experiments in a wave flume, at the first stage, the leakage of sand starts (Figure 2a), then the generation of caves observed (Figure 2b). Enlargement of the caves continues until

\footnotetext{
${ }^{1}$ Department of Civil Engineering, The University of Tokyo, 7-3-1, Hongo, Tokyo, 113-8656, Japan
} 
the soil cannot support the weight of soil above developed cave (Figure 2c). Consequently, the collapse of the pavement takes place (Figure 2d). Bierawski et al. (2002) noted that the backfill leaks from behind a seawall due to pressure oscillations. Also, they noted that leakage of the backfill starts very slowly and leads to failures in the seawalls. Nakamura et al. (2008) have also reported that the leakage of the backfill phenomenon is closely related to the wave-induced pressure response. Later, Hur et al. (2007) studied the sand suction mechanism. Findings from the experiments agreed with Takahashi et al. (1996). A similar mechanism has been described for the destruction of the subsidence of backfill.
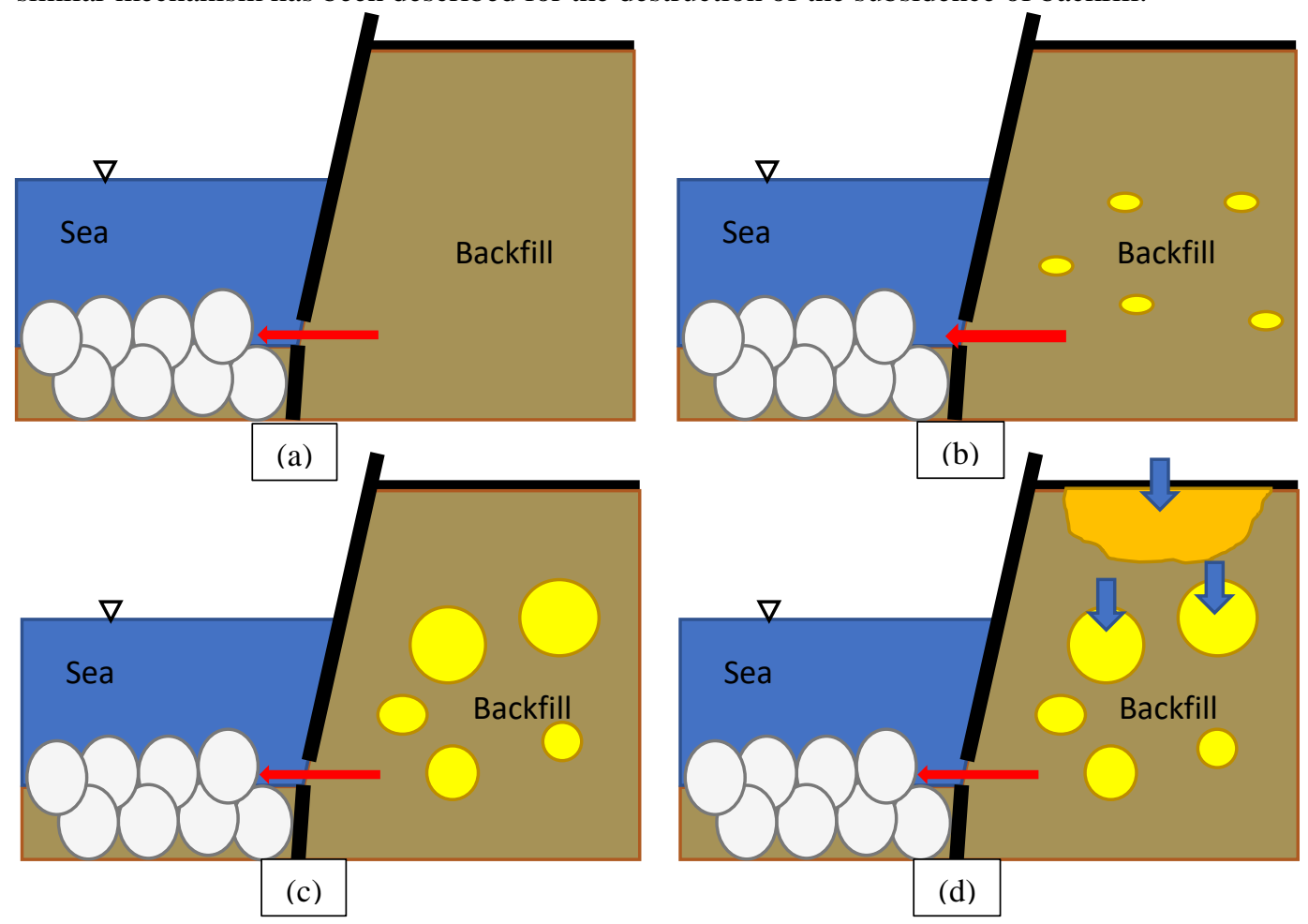

Figure 2 Stages that leads to subsidence of backfill surface

Previous experimental studies mostly considered the final result of a scaled hydraulic model under the exposure of a sequence of waves. However, in this study, the movement of soil during one cycle is also examined with video analysis.

In this study, the main objective is to identify the void expansion mechanism (Figure 2c) after a void developed inside the backfill of the seawall (Figure 2b) due to the leakage of the backfill towards the seaside. In order to confirm the acceleration of void development, it is essential to check whether the rate of leakage is proportional to the void size. The sand leakage phenomenon is closely related to the waveinduced seabed response. As wave pressure propagates in the soil medium, pore pressure and effective stresses change and cause strains in the soil. The difference in pressures at water-soil interface may generate the leakage of backfill material through imperfections in the geotextile materials, filter layer or from the foundation. As stated in the previous studies (Sumer, 2014), the presence of air in seabed causes high-pressure gradients that may accelerate void development. Another mechanism that may be responsible for the leakage of sand is the displacement of the soil near the gap by pressure fluctuations. Laboratory experiments have been performed in order to examine the sand leaking process.

\section{EXPERIMENTS}

Laboratory experiments have been conducted in an oscillatory flow tunnel (OFT) at the University of Tokyo. Schematic view of the OFT is given in Figure 3. The test section is $600 \mathrm{~cm}$ long $7 \mathrm{~cm}$ wide and $28 \mathrm{~cm}$ deep and one side is made up of glass to observe experimental developments inside the OFT. Back and forth motion of the piston generates the flow inside the tunnel under pressurized environment.

The flow characteristics are controlled with the piston movement. The displacement and the period of the piston can be adjusted. As the piston and test section dimensions are known, horizontal displacement amplitude, $a$ in the test section can be found. Thus, the water particle displacement is calculated as, 

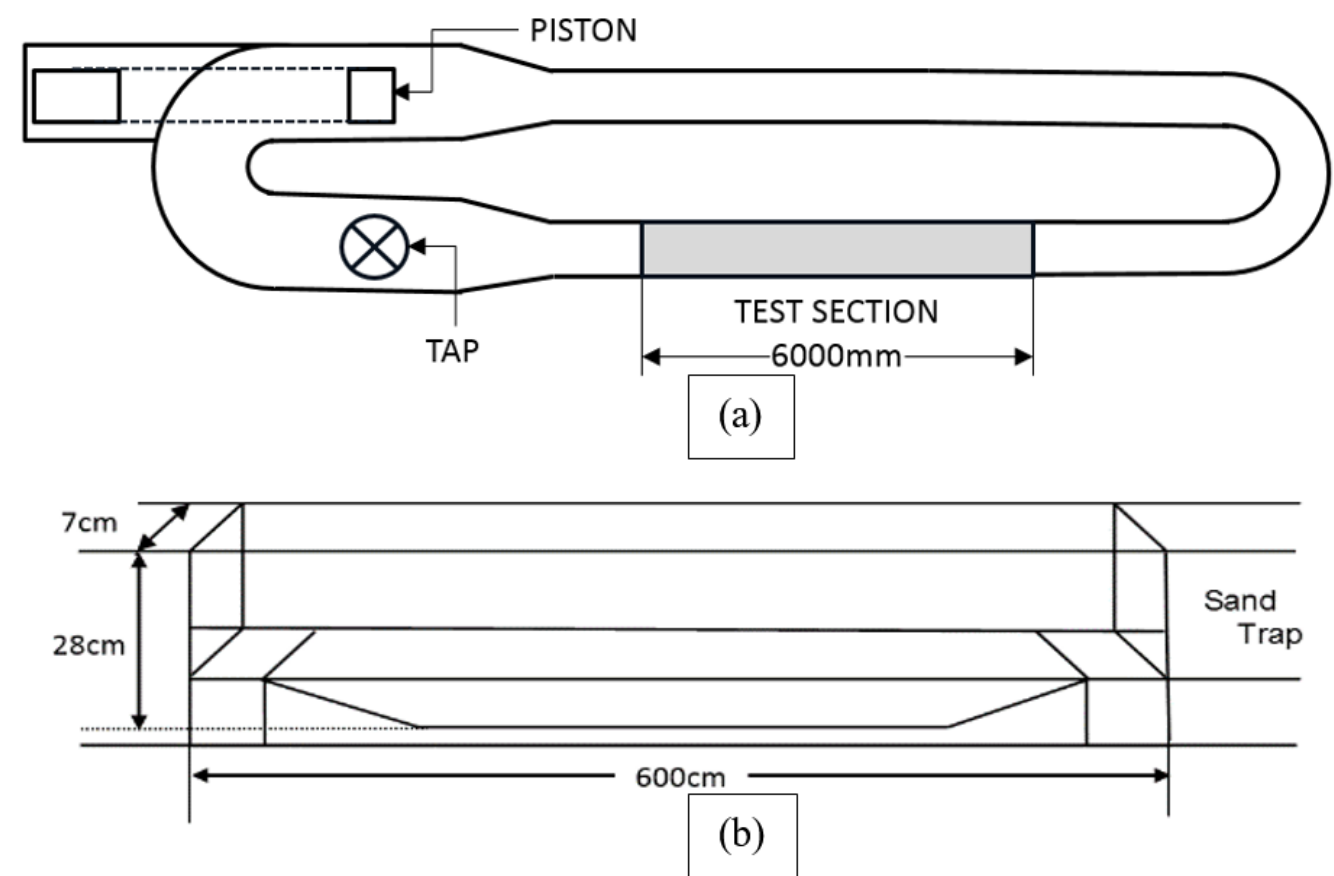

Figure 3 Schematic view of the Oscillatory Flow Tunnel. (a) is the top view, (b) is test section from the side

$$
\xi=a \cos \omega t
$$

where $\omega$ is the angular frequency and $t$ is time. Then, the velocity is calculated as,

$$
u=\frac{\partial \xi}{\partial t}=-a \omega \sin \omega t
$$

so, inside the test section, the velocity is uniform everywhere Later, the pressure is calculated from the relationship with velocity,

$$
\begin{gathered}
\rho \frac{\partial u}{\partial t}=-\frac{\partial p}{\partial x} \\
p=\rho a \omega^{2}\left(x-x_{0}\right) \cos \omega t
\end{gathered}
$$

where $\rho$ is the density of water, $x$ is the desired location where pressure is needed and $x_{0}$ is the location where the pressure fluctuation becomes zero. The pressure amplitude changes linearly throughout the test section. It is highest at the left end and lowest at the right end of the test section.

To simulate the leakage through a small gap like the real seawall environment, a small acrylic rectangular parallelepiped-shaped container with a small gap on the top has been prepared. The container is $30 \mathrm{~cm}$ long, $6 \mathrm{~cm}$ wide and $8 \mathrm{~cm}$ deep.

As one of the purposes of this study is to examine the effect of voids filled by air on the leakage rate, air has been introduced to the soil medium with small toy balloons (Figure $4 \mathrm{~b}$ ) to simulate the voids in the backfill of a seawall. They have been prepared by placing $40 \mathrm{ml}$ of air and sealing carefully to have similar compressibility in each balloon. Two types of sand have been used in the experiments. They are named as fine grain $\left(\mathrm{d}_{50}=0.25 \mathrm{~mm}\right)$ and coarse grain $\left(\mathrm{d}_{50}=0.89 \mathrm{~mm}\right)$

\section{Preparation of the Experiments}

Firstly, the balloons are placed to the bottom of the acrylic container. Then, sand is filled with hitting from the sides to have similar porosity in each experiment. Then, the top of the container is closed with two acrylic plates just providing a small gap on top. Afterward, the plates are attached to the container with waterproof tape to make sure the only the flow on top of the small gap affects the soil medium. As 
the plates are rather rigid, the only application of flow occurs through the small gap. Then, the container is placed under water to make the sure inside the container there is no air except the balloons.
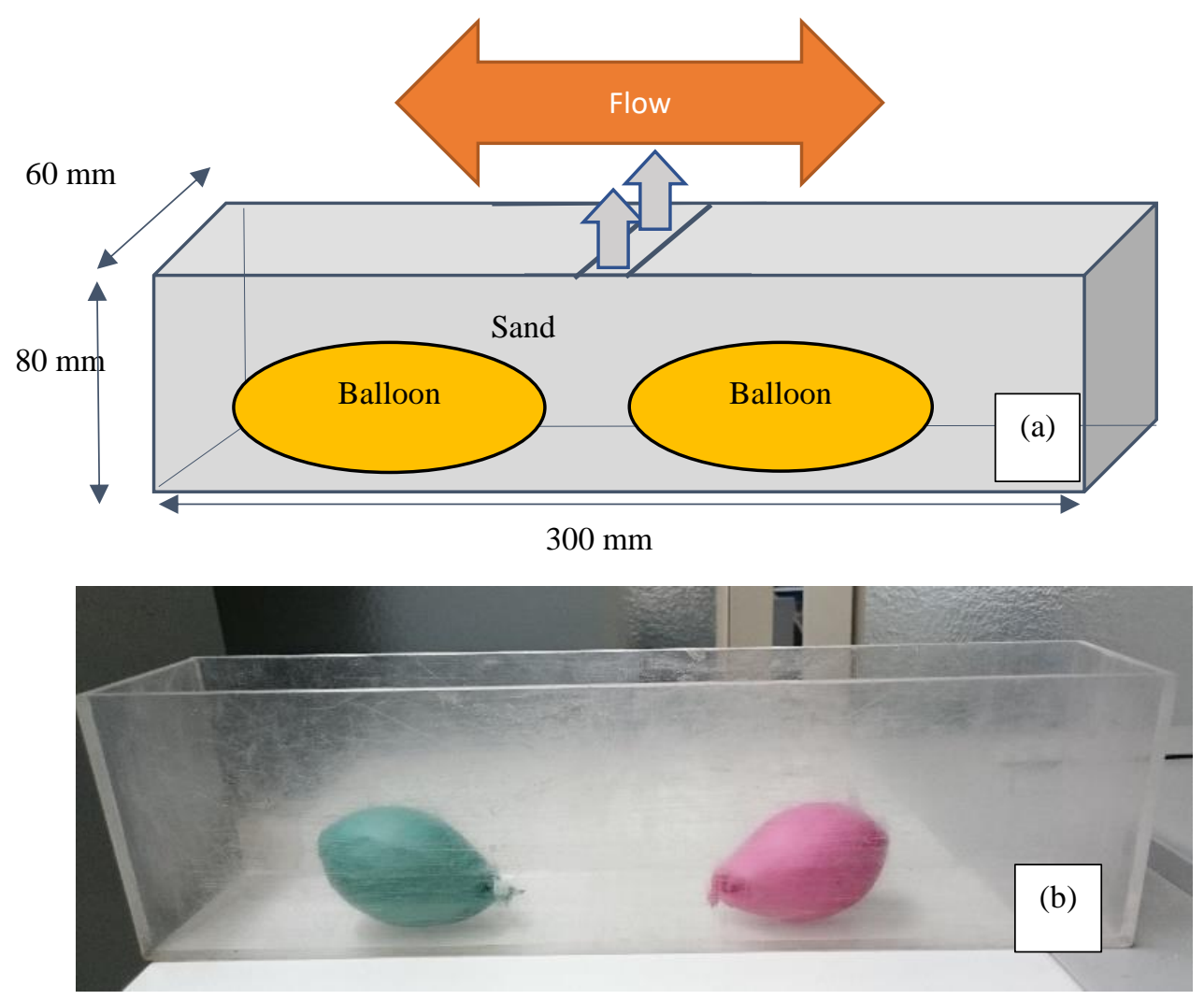

Figure 4 Schematic view of the experiment (a) and photograph of acrylic container and balloons (b)

Later, the container is put to the bottom of the OFT, which is leveled beforehand for the container to have a smooth horizontal flow on top of it without any eddies. After that, the OFT is filled with water and the lids are closed. Under pressurized environment, the experiment is started with setting up the period and the horizontal displacement of the piston, which creates the oscillatory flow inside the tunnel. Sand leakage through the small gap under oscillatory flow (Figure 4a) is observed and recorded with video cameras. After several experiments, it has been realized that 3 minutes later, the soil profile reaches a stable condition. Therefore, the oscillatory flow has been applied for 3 minutes for each experiment.

Since the weight of leaked sand is very limited, weighing the container before and after the experiments is prone to error. Therefore, scoured portion of soil is considered as the leaked sand from the container. It has been evaluated with the help of images taken during the experiments. Firstly, the scoured area in $\pm 3 \mathrm{~cm}$ horizontal direction is selected. Then, the number of pixels in the image for the scoured area are counted through image processing. Scoured area, $A_{s}$, after the experiment is taken as the leaked sand amount. Also, maximum scoured depth has been calculated with a similar manner. Pixel count at the deepest scour location is scaled and recognized as the maximum scour depth, $d_{s}$ (Figure 5). 


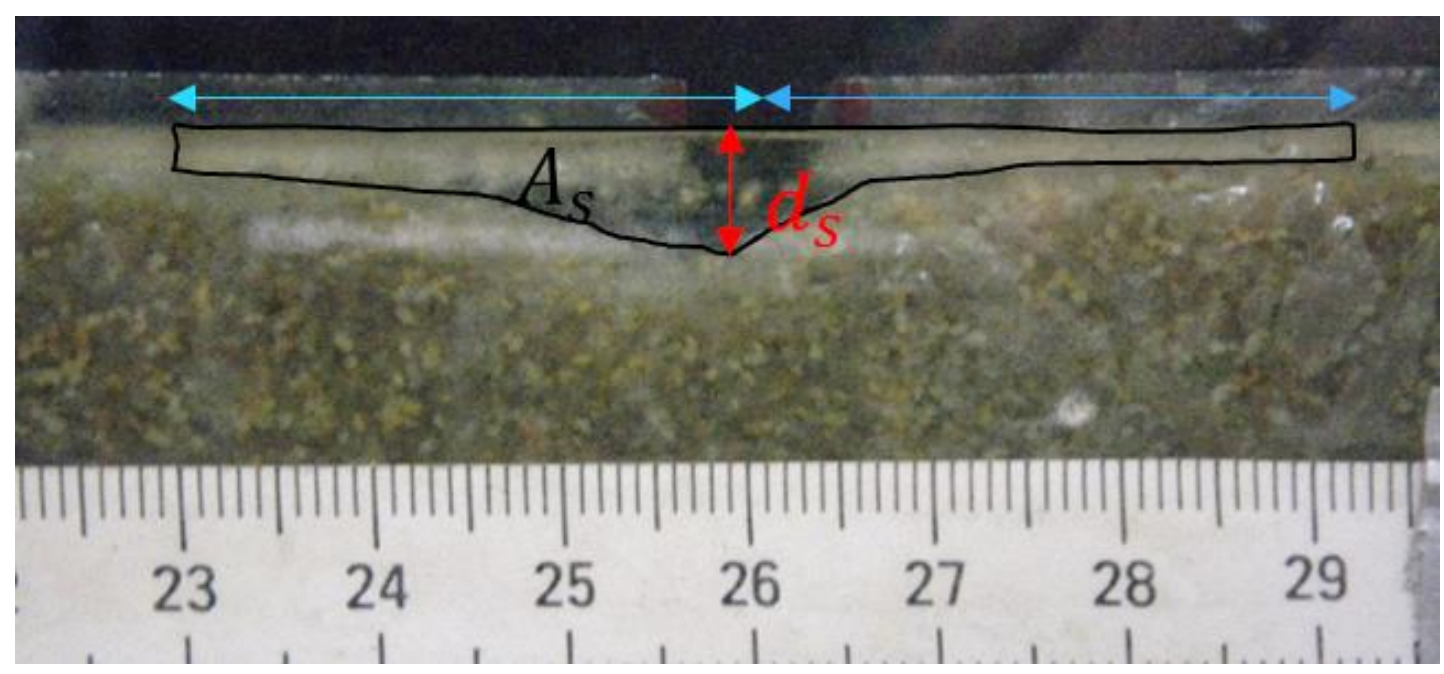

Figure 5 Scoured area and maximum scoured depth

\section{RESULTS}

To measure the pressure amplitudes, manometers have been placed along the OFT. According to the Figure 6, the pressure amplitude decreases as the manometer goes to the right from the left for 4 different experiments. This graph matches well with the calculation for pressure theoretically in Eq. 4, where it is suggested that the pressure will decrease along the OFT. Note that the velocity of the flow is same at every location for the same experiment.

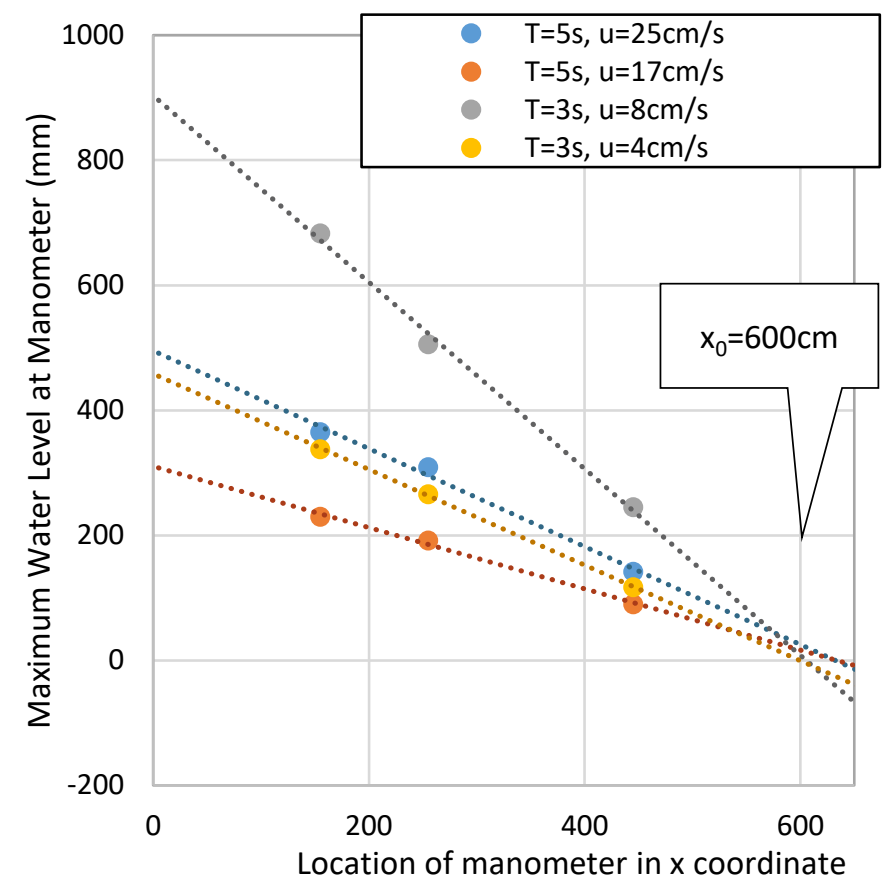

$(\mathrm{cm})$

Figure 6 Measurement of pressure oscillations along the oscillatory flow tunnel

Then, the container has been placed at various locations along the OFT under same flow conditions. Five experiments revealed that the measured leakage decreased as the container placed towards the right side where the pressure oscillation decreases despite the flow velocity remains same (Figure 7). 


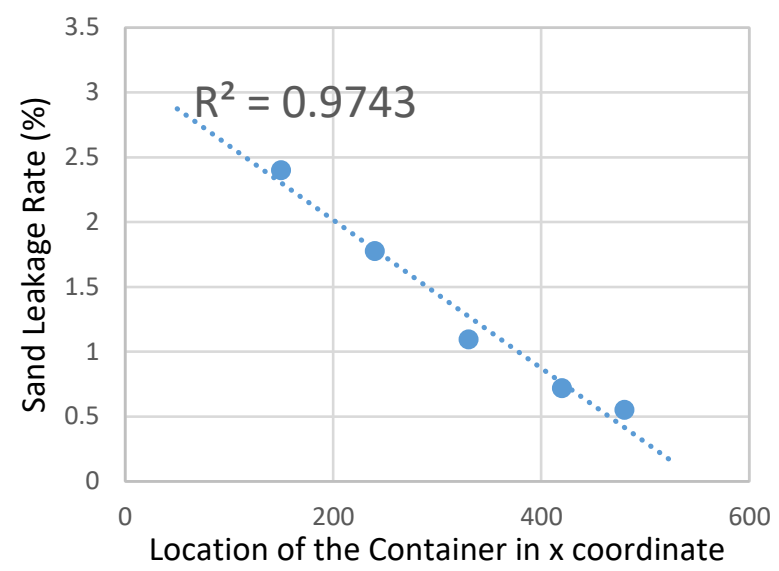

$(\mathrm{cm})$

Figure 7 Sand leakage rate along the oscillatory flow tunnel

Furthermore, the frame-by-frame analysis of the video revealed that the leakage is highest during the pressure is minimum and velocity is near zero. Figure 8 shows the images from the experiments while the flow is towards the right side. The velocity is negative and the pressure is decreasing to minimum from maximum. At this time sequence, the soil medium gets closer to the gap and at the end when the pressure is minimum a high leakage is observed.
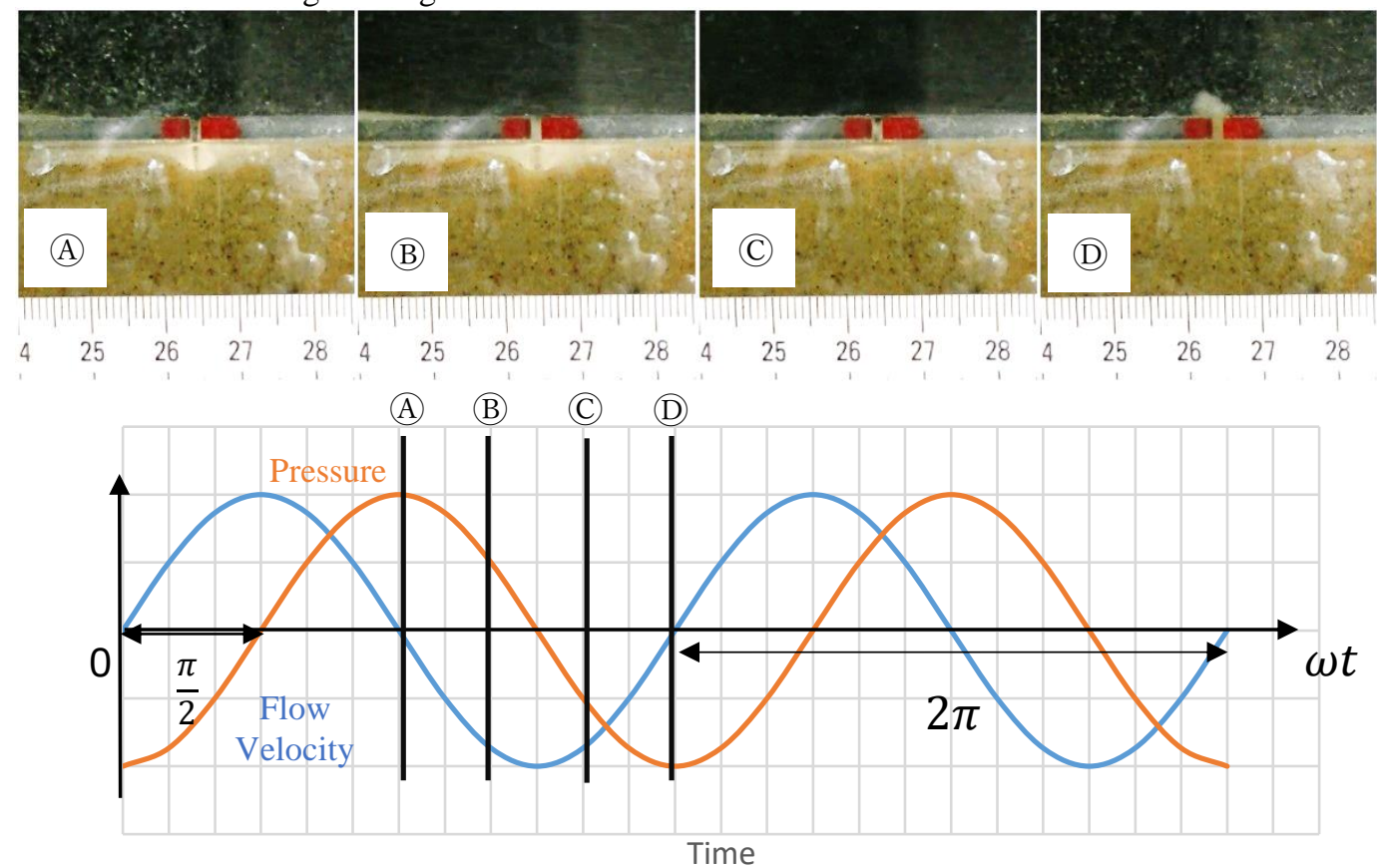

Figure 8 Recordings from experiments while the flow is towards right (velocity is negative)

Figure 9 shows the images from the experiments while the flow is towards the left side. The velocity is positive and the pressure is increasing to maximum from minimum. At this time sequence, the soil medium goes down, away from the gap by the effect of pressure increase. Also, when the pressure is above zero, very low leakage has been observed. 

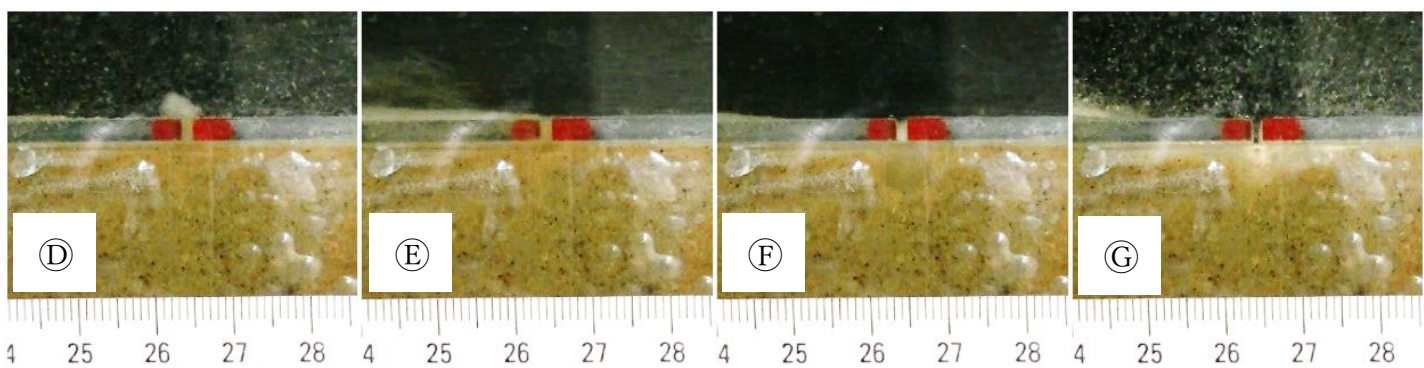

(D)

(E)

(F) (G)

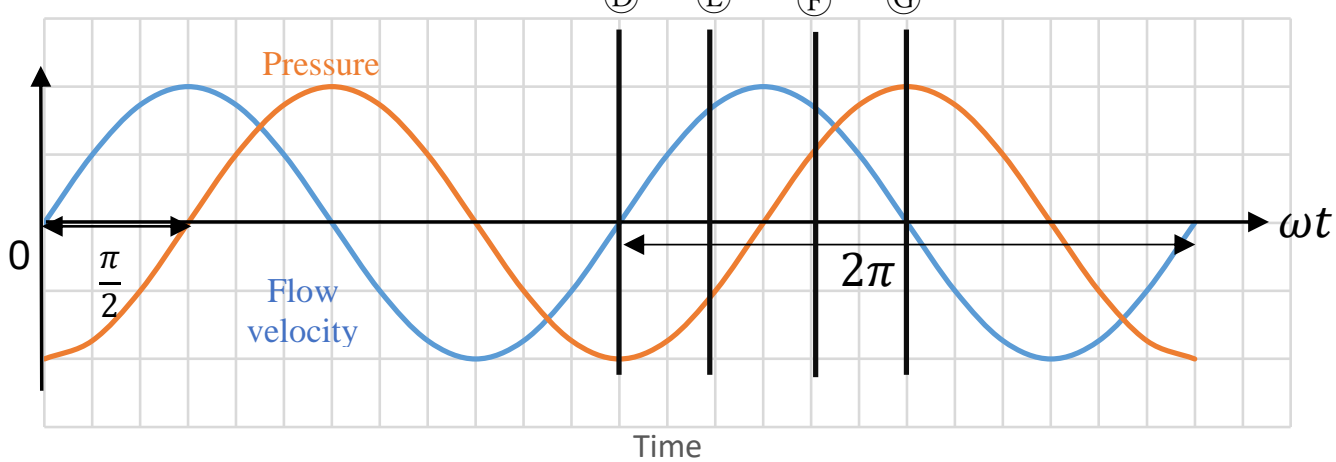

Figure 9 Recordings from experiments while flow is towards left (velocity is positive)

Additionally, an experiment with two small pressure sensors has been carried out. One pressure sensor has been placed on top of the container near the gap, and the other one has been placed inside a toy balloon. Figure 10 shows the measurements between 20-40 seconds from this experiment.

According to the result, damping in the pressure amplitude and a slight time lag have been observed. Pressure amplitude has attenuated around 20-30\%. And, around 10 degrees of phase lag have been noticed.

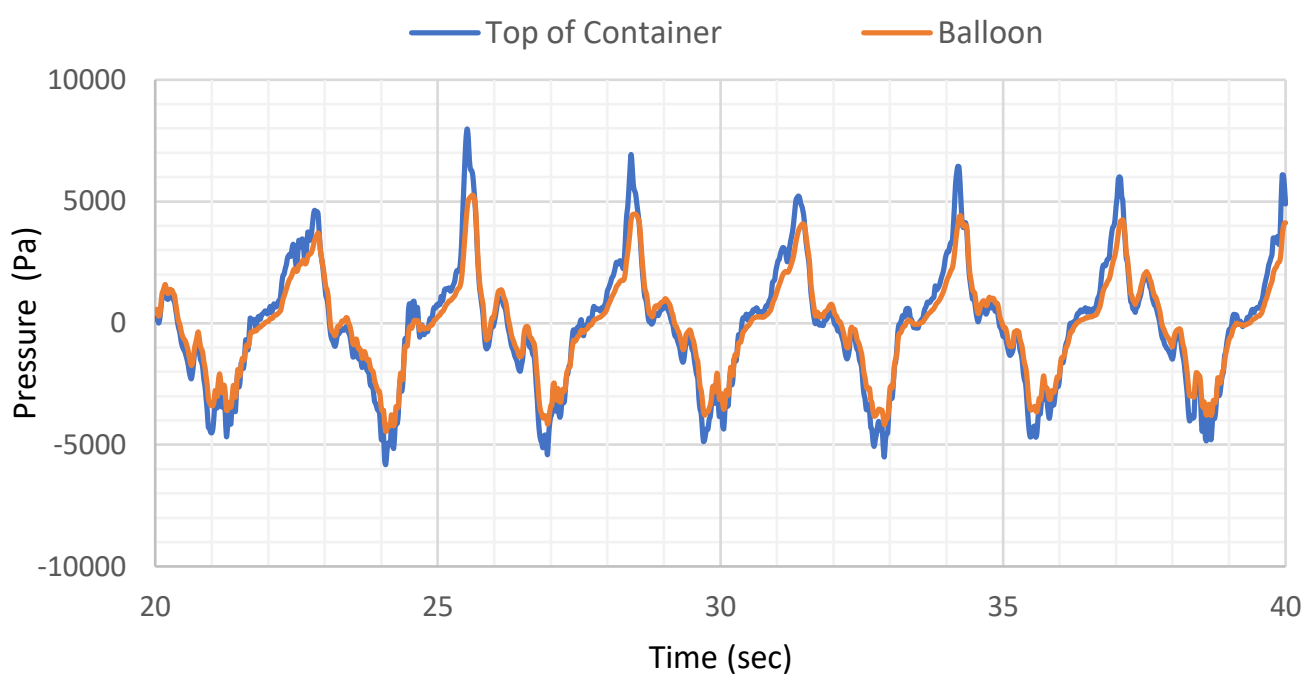

Figure 10 Pressure oscillations on top of the container and inside a balloon

Later, the sensitivity analysis on the amount of air placed in soil medium has been performed. Fundamentally, inserting balloons changes the compressibility of the soil medium. So, this analysis can be seen also as the effect of the compressibility on the leakage of soil. As soil grains and water are incompressible comparing with the air, putting more air will increase the overall compressibility of the soil medium. Previous studies on wave-induced soil response (Jeng 2013, Sumer 2014) reveal that the pressure response is getting poorer when the compressibility increases. It is because the pressure on the 
soil surface dissipates rather rapidly near the upper region of soil because air mitigates the pressure by expansion or contraction. Thus, in the light of this background, results from trials with 4 balloons (160 $\mathrm{ml}), 2$ balloons $(80 \mathrm{ml})$ and no balloon are given in Figure 11.

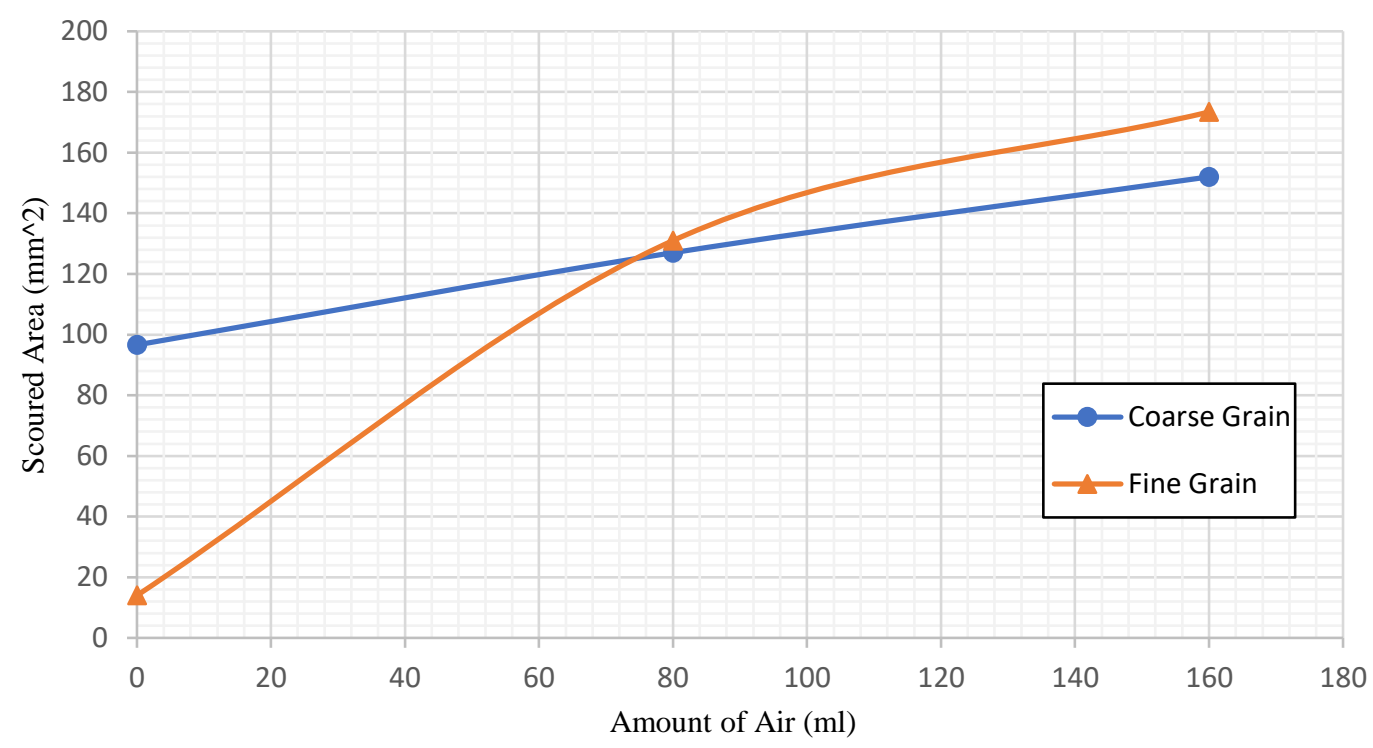

Figure 11 Scoured area depending on the amount of air inside soil medium for fine and coarse grain soil

However, it is noted that for the coarse grain sand, even at the beginning, some scour can be observed. It is because filling of the container cannot be performed fully due to large grains. Therefore, a new parameter, relative scour has been introduced. It is calculated as the scour divided by the scour when there is no air.

$$
A_{s r}=\frac{A_{s}}{A_{s 0}}=\frac{\text { Scour }}{\text { Scour for (no - air) }}
$$

The relationship of the relative scour and the amount of air for both fine grain and coarse grain is given in Figure 12. Note that, in this figure, the relative scour crosses the vertical axis at the value of 1 . According to this figure, the sand leakage rate is proportional to the air amount in the soil medium. Introducing more air into the container increases the rate of leakage. Already stated acceleration mechanism of void development has been confirmed with this result. Especially, in the fine grain soil, the sand leakage rate is influenced more from the air amount. Though there is a slight increase, this effect is not conspicuous in coarse grain sand. There can be several reasons for this outcome. As the movement of soil grains out of the container is related to the pressure gradient between soil inside the container and the flow above the container, the pressure gradient in fine grain soil is much higher than the coarse grain. According to Jeng (2013), the pressure response of fine grain soil with some air presence in the soil medium is worse than the coarse grain soil. In other words, the pressure gradient generated in fine grain soil is much higher than the coarse grain soil. Another reason might be the generated force on soil grains cannot lift the coarse grain soil. Therefore, the leakage of sand in the coarse grain sand is not as much as the leakage in fine grain soil. 


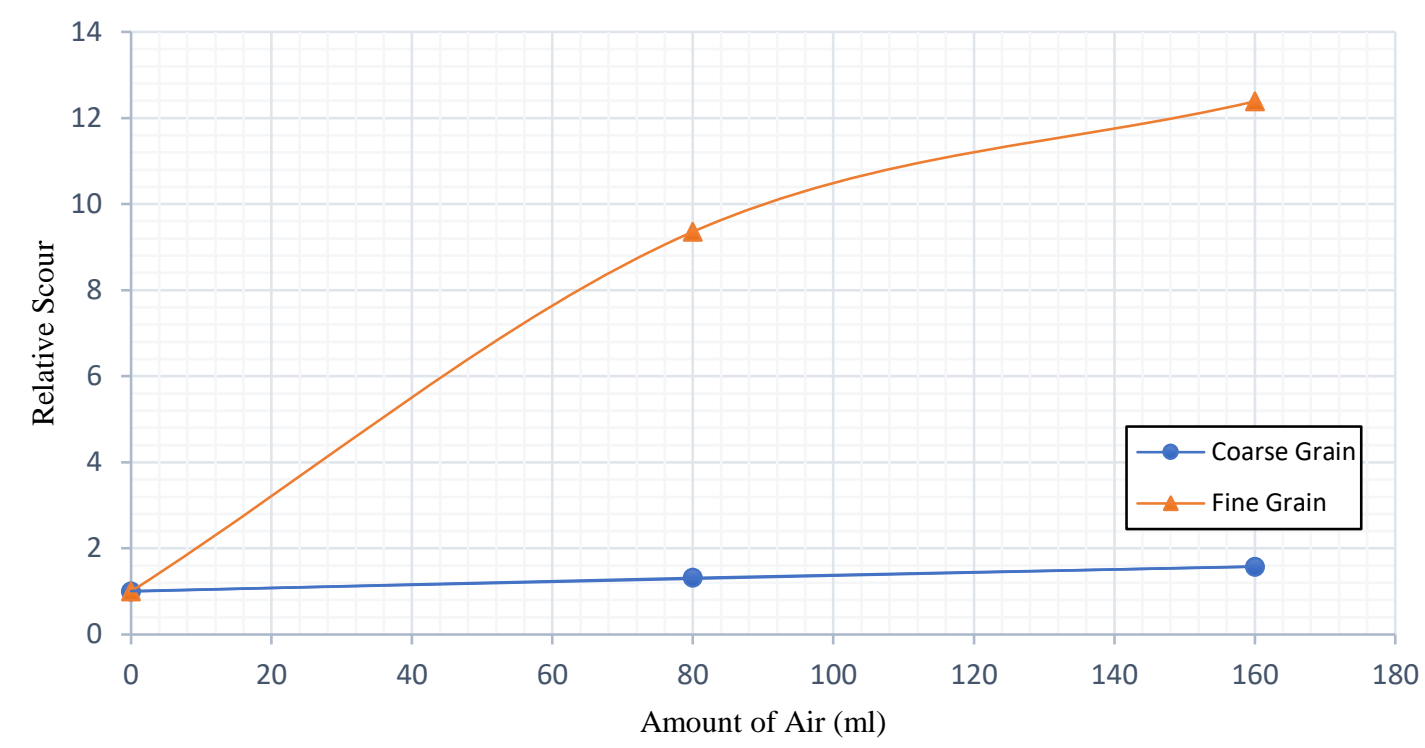

Figure 12 Relative scour depending on the amount of air inside soil medium for fine and coarse grain soil

The sand leakage depending on the gap width with the fine grain soil experiments are given in Figure 13. 4 balloons have been placed to the bottom of the container which is filled with fine grain sand. As gap width increases, the point of application of the pressure oscillations and the gap from which the sand leaks change.

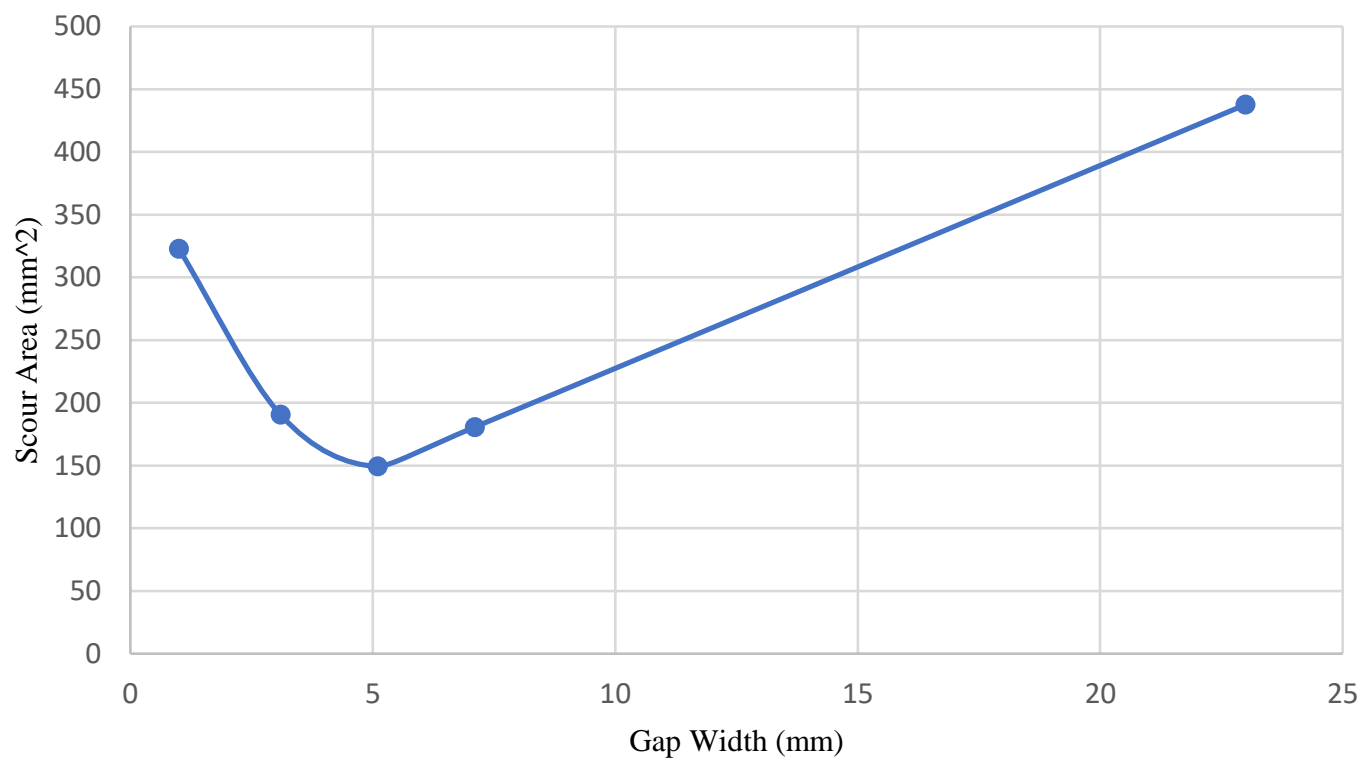

Figure 13 Scoured area depending on the gap width ( 4 balloons- fine grain soil)

While the gap gets wider, the leakage amount would be expected to increase owing to the shear stress and regular sediment transport processes. Therefore, the experiment with $23 \mathrm{~mm}$ gap caused a large amount of leakage due to large exposed area to shear stress. However, when the gap is very small $(1 \mathrm{~mm})$, it is observed that the leakage amount is also very high. The reason behind this might be explained with the poor pressure transmission when the gap is very small. As explained before, poor pressure transmission leads to high-pressure gradient and intensify the amount of leakage. However, as the gap gets wider, the pressure response becomes better and shear stress turns into more effective in the leakage of sand. It is also worth noting that from the experiments of Takahashi et al. (1996), when the gap is too small, it may get blocked by the soil grains and no leakage can be observed. Although in the 
current experiments, blockage of the gap has not been observed, it can happen in a real seawall environment.

\section{DISCUSSION OF RESULTS}

In the current laboratory experiments, a larger void appeared to accelerate further enlargement of the void. One of the reasons for the large leakage under large void is the pressure gradient between soil medium and the water flow enhances the leakage of the soil particles with enlarged inertia and drag forces. High-pressure gradient results in high sand leakage rate and the pressure gradient depends on the soil response.

Another reason is due to the high compressibility of the soil medium including large void, the displacement of soil particles near the gap is larger when air present in the soil. The enlarged displacement may also contribute to the high leakage rate of sand while the amount of air is large.

These reasons reveal that as the air amount increases in the soil medium, the rate of leakage of the soil grains also increases. In other words, the sand leakage rate is proportional to the air amount in the soil medium. As the void develops with the leakage, void development in soil is exponential based on the following equations:

$$
\begin{aligned}
& \frac{d V_{\text {air }}}{d t}=\alpha V_{\text {air }} \\
& \mathrm{V}_{\mathrm{air}}=c e^{\alpha t}
\end{aligned}
$$

where the $\alpha$ and $c$ are relevant coefficients.

As the behavior of soil change depending on the type of air presence, it is important to know in the coastal environment which type of air present. Sumer (2014) reported that in the sea environment, air is generally in the shape of bubbles which are much larger than the grain size of soil particles. Therefore, the experimental setup in this study may provide a soil medium which has mechanics like real sea environment.

The soil permeability is a measure of how rapidly fluid is transmitted between grains. Also, it is generally related to the grain size of the soil particles for sands. In coarse grain soil (high permeability), the pressure gradient is rather low. In other words, pressure transmission is better in coarse grain soil. Therefore, as observed in the experiments, the rate of leakage with coarse grain soil has not been affected by the placement of balloons as much as fine grain soil. As stated in Jeng (2013), under the same degree of saturation, pressure gradient near the soil surface is smaller for more permeable material. This explains the effect of air balloons in the coarse sand is not as much as the fine sand.

One important shortcoming of these experiments should be mentioned. In these experiments, gravity acts as resisting force. However, in a real seawall environment, probably gravity will act as a driving force. However, rather than the estimating the sand leakage rate, the main purpose is to reveal the acceleration mechanism. Therefore, the experiments served to this purpose.

Another concern about this experiments is the location of the hole which causes the leakage of sand in the seawall is also very important. If the hole is located near the mean sea level, then the leakage may depend on also the wave velocities and breaking characteristics rather than just pressure oscillations. However, if the hole is in relatively deep water or protected from direct wave attack by the armor stones, the main driving force can be the pressure oscillations.

Field study might be necessary to fully understand the mechanism of void development in seawalls. Since the laboratory experiments are affected by the scale of the forces generated. At the laboratory, the used soil is same that is found in the field. However, in a laboratory environment, the forces are not as much as in the field. Furthermore, the behavior of sand leakage largely dependent on the compressibility of the soil which is almost impossible to achieve to reach in the laboratory. However, it is hard to carry out a field study for this subject as the location of the subsidence of a backfill is not known before the failure. Also, the amount of time necessary to cause such destruction is not clear.

\section{CONCLUSION}

Laboratory experiments have confirmed the acceleration mechanism of the void development in the backfill of a seawall. This mechanism is due to both the pressure response of the soil and strains near the gap as the backfill gets more compressible by air replacement instead of soil grains in the backfill. The leakage of backfill may start very slowly. However, due to the leaking sand, it will become more compressible and accelerated void development may cause various failures in seawalls. 
The leakage of sand through a small gap has been found out to be closely related to the upward seepage flow due to the pressure gradient between soil medium and water flow. Therefore, the response of the soil medium to the pressure oscillations is the key in this process. If soil response to pressure fluctuation decreases, it is likely to have a greater soil leakage.

Compressibility and permeability of soil greatly influence the response to pressure. From compressibility point of view, the introduction of air balloons interrupts the pressure propagation and leads high-pressure gradients. In return, higher drag and inertia forces generate higher leakage.

The permeability of the material has also a significant effect on seabed response. More permeable materials transfer the pressure easily. So, increase in permeability will also increase the pressure response and decrease the rate of leakage.

In this study, poor response of the soil leads higher leakage amount. However, a good pressure response can be also dangerous for the both structure and the backfill, so it is important to lower the scale of pressure oscillations before hitting to backfill by putting armor stones or toe protection.

With all these results, it is important that the leakage should be prevented. Even small amount of leakage can rapidly increase the discharge of the backfill towards the seaside. Therefore, careful inspection and a quick countermeasure are important especially at the initial stage of void development.

\section{REFERENCES}

Bierawski, L. G., Maeno, S., Gotoh, H., \& Harada, E. (2002). DEM-FEM model of the outflow of backfilling sand from behind a seawall under wave motion. In Proc., 5th Int. Conf. on Hydro-Science and Engineering.

Hur, D. S., Nakamura, T., \& Mizutani, N. (2007). Sand suction mechanism in artificial beach composed of rubble mound breakwater and reclaimed sand area. Ocean Engineering, 34(8-9), 1104-1119.

Jeng (2013): Porous Models for Wave-seabed Interactions, Springer, Heidelberg, Germany, pp. 62-63.

Nakamura, T., Hur, D. S., \& Mizutani, N. (2008). Mechanism of backfilling sand discharge from a gap under vertical revetment. Journal of waterway, port, coastal, and ocean engineering, 134(3), 178186.

Sumer (2014): Liquefaction around Marine Structures, World Scientific, New Jersey, pp. 7-9

Takahashi, S., Suzuki, K., Tokubuchi, K., \& Shimosako, K. I. (1996). Experimental Analysis of the Settlement Failure Mechanism Shown by Caisson-Type Seawalls. Coastal Engineering Proceedings, $1(25)$.

Zonguldak'ta deniz kıyısındaki kaldırım ve yol çöktü. (2015, 6 25). Retrieved from CNN TURK: http://www.cnnturk.com/ 